\title{
Magnetic resonance imaging-monitored conservative management of traumatic spinal epidural hematomas
}

Giovanni La Rosa, M.D., Domenico d'Avella, M.D., Alfredo Conti, M.D., Salvatore Cardali, M.D., Domenico La Torre, M.D., Fabio Cacciola, M.D., Marcello Longo, M.D., and Francesco Tomasello, M.D.

Department of Neurosurgery, and Service of Neuroradiology, University of Messina School of Medicine, Messina, Italy

Spinal epidural hematomas (SEHs) are uncommon complications caused by traumatic injuries to the spine. Emergency surgical evacuation is the standard treatment. Although recognized in the literature, the possibility of nonsurgical treatment of traumatic SEHs is far from being codified. The authors report on the treatment of four patients whose traumatic SEHs were diagnosed by magnetic resonance (MRI) imaging and managed conservatively with excellent results.

All patients had suffered severe spine injury with fracture of a lumbar vertebral body, were admitted within 12 hours of trauma, and exhibited only minimal neurological disturbances on admission. Magnetic resonance imaging studies were performed within 24 hours of trauma. Hematomas appeared isointense/slightly hyperintense on $\mathrm{T}_{1}$ - and heterogeneous on $\mathrm{T}_{2}$-weighted $\mathrm{MR}$ images. Clot thickness varied between $0.8 \mathrm{~cm}$ and $1 \mathrm{~cm}$, width between $1 \mathrm{~cm}$ and $1.8 \mathrm{~cm}$, and length between 2.7 and $9 \mathrm{~cm}$. In light of each patient's fairly good neurological condition a conservative approach was taken. In all cases serial MR imaging documented progressive clot resolution, which was completed within 8 to 10 days of trauma. At discharge all patients were neurologically intact.

The conservative treatment option of traumatic SEH should be reserved for exceptional cases whose deficits are minimal, when neurological deterioration is followed by early and sustained spontaneous recovery, and when there are clear medical contraindications for surgery. The results of the present study confirm that nonsurgical treatment is feasible in a subgroup of minimally symptomatic patients who harbor moderate-sized SEHs. Although the authors' experience shows a good spontaneous outcome of some traumatic SEH, further studies are necessary to understand the real spectrum of nonsurgical treatment of such lesions.

Key Words * conservative management * magnetic resonance imaging * spinal cord injury * spinal epidural hematoma

Spinal epidural haematomas (SEHs) are a well-recognized but uncommon complication of traumatic injuries to the spine.[2,7] They are classically considered neurosurgical emergencies because of the 
hazard of progressive cord compression that results in a permanent neurological deficit.[8,11,15] However, an increasing number of nonsurgically managed "spontaneously" resolved SEHs reported in more recent literature suggests that surgical treatment may be unnecessary when the patient's neurological status is stable or improving, and that SEHs in a number of patients may resolve spontaneously, with the patient making a complete neurological recovery.[3,12,17,18,20,21] The question then arises of whether a conservative treatment might be safely adopted in selected cases of posttraumatic SEH as well. Although a few reports of spontaneous healing of traumatic SEH have appeared in the literature, $[4,13,19]$ the possibility of nonsurgical treatment is far from being codified. We report our experience in four patients whose posttraumatic SEHs were diagnosed by MR imaging and managed conservatively with excellent results.

\section{CLINICAL MATERIAL AND METHODS}

This case series includes four patients with posttraumatic SEH who were seen between January 1996 and April 1998 at the Department of Neurosurgery of the University of Messina Medical School, an academic neurosurgical unit which also serves as referral center for spine surgery in the region. All patients had suffered severe spine cord injury with associated vertebral fracture, and were admitted and evaluated within 12 hours of the traumatic event. Neurological function was evaluated using the five-tier Frankel grading scale.[9] Demographic, clinical, and neuroradiological data (including electromyography, spine x-ray films, spinal computerized tomographic and magnetic resonance [MR] studies) were gathered retrospectively by performing an analysis of patient records, as summarized in Table 1. Patients underwent clinical evaluation at followup approximately 6 months posttrauma.

\begin{tabular}{|c|c|c|c|c|}
\hline SUMMARY OF CLI & QL AND NEURORADIOL & $\begin{array}{l}\text { TABLE } 1 \\
\text { CAL FIND NGS IN } \\
\text { D CONSERYATW }\end{array}$ & R PATIENTS W & TRAUMATIC SEHS \\
\hline Characteristic & Case 1 & Case 2 & Case 3 & Case 4 \\
\hline $\begin{array}{l}\text { age (yrs) } \\
\text { hematoma (cm) }\end{array}$ & 32 & 20 & 50 & 27 \\
\hline $\begin{array}{l}\text { length } \\
\text { width } \\
\text { thickness }\end{array}$ & $\begin{array}{l}2.7 \\
1 \\
0.8\end{array}$ & $\begin{array}{l}3.6 \\
1 \\
0.5\end{array}$ & $\begin{array}{l}9 \\
1.8 \\
0.8\end{array}$ & $\begin{array}{l}6.5 \\
1.7 \\
1\end{array}$ \\
\hline symptoms \& signs & $\begin{array}{l}\text { radicular pain ti- } \\
\text { lat radicular sen- } \\
\text { sory defidt at L-1 }\end{array}$ & radicular pain & radicular pain & $\begin{array}{l}\text { radicular pain uni- } \\
\text { lat radicular mo- } \\
\text { tor deficit at L-4 }\end{array}$ \\
\hline $\begin{array}{l}\text { extension level } \\
\text { location } \\
\text { gracture le vel } \\
\text { resolution time } \\
\text { (days) }\end{array}$ & $\begin{array}{l}\mathrm{L}-1 \\
\text { anterior to sac } \\
\mathrm{L}-1 \\
9\end{array}$ & $\begin{array}{l}\mathrm{L} 1-2 \\
\text { anterior } \\
\mathrm{L}-1 \\
8\end{array}$ & $\begin{array}{l}\text { T11-L2 } \\
\text { anterior } \\
\mathrm{L}-1 \\
10\end{array}$ & $\begin{array}{l}\mathrm{L} 2-4 \\
\text { anterolateral } \\
\mathrm{L}-3 \\
10\end{array}$ \\
\hline
\end{tabular}

The investigational neuroradiological MR imaging protocol was begun in all patients within 24 hours posttrauma. A 1.5 Tesla unit (Siemens Magnetom, Erlangen, Germany) was used. The imaging protocol consisted of $\mathrm{T}_{1}$-weighted (TR $500 \mathrm{msec}$, TE $12 \mathrm{msec}$, matrix 256 x 256, thickness 3-4 mm) and proton density $\mathrm{T}_{2}$-weighted double echo-spin echo sequences (TR $2200 \mathrm{msec}$, TE 20/80 msec, matrix 256 x 256, thickness 3-4 mm) in the sagittal plane; $\mathrm{T}_{2}$-weighted high-resolution images (TR $5400 \mathrm{msec}$, TE 99 msec, matrix $300 \times 512$, thickness $4 \mathrm{~mm}$ ) in the transverse plane; $\mathrm{T}_{2}$ turbo spin echo-train length images (TR $2800 \mathrm{msec}$, TE $1100 \mathrm{msec}$, flip angle $150^{\circ}$, thickness $50 \mathrm{~mm}$ ) for myelographic effect; and 
gradient-echo $\mathrm{T}_{2}$-weighted images (TR $540 \mathrm{msec}$, TE $15 \mathrm{msec}$, flip angle $15^{\circ}$, thickness $4 \mathrm{~mm}$ ). Serial MR control studies were then performed in all patients at 3 and 8 to 10 days posttrauma.

\section{RESULTS}

Four patient with posttraumatic spinal SEHs were identified. They were all male. The average age of the patients was 35 years (range 20-50 years). The cause of trauma in all patients was a low-height fall that caused vertebral body fractures at high lumbar level (L-1 in three cases and L-3 in one case), with integrity of the vertebral canal and absence of vertebral dislocation. None of these patients had a positive history for anticoagulation or clotting disorders. In all patients pain at the site of the lesion was the initial symptom. True radicular pain was present in all patients at the initial neurological examination, performed within 12 hours of the traumatic event. Two neurologically intact patients were designated Frankel Grade E, and two mildly symptomatic patients with isolated radicular signs were designated Frankel Grade D. No patient exhibited evidence of bladder dysfunction. In all patients MR imaging of the spine demonstrated an epidural hematoma of moderate size, correctly identifying the dorsoventral and craniocaudal extension of the clot, as summarized in Table 1. Hematomas displayed the typical signal characteristics pattern,[19] appearing in the acute phase as isointense or slightly hyperintense on $\mathrm{T}_{1}$ - and heterogeneous on $\mathrm{T}_{2}$-weighted images biconvex-shaped lesions. Clot thickness varied between $0.8 \mathrm{~cm}$ and $1 \mathrm{~cm}$, with widths between $1 \mathrm{~cm}$ and $1.8 \mathrm{~cm}$ and lengths between $2.7 \mathrm{~cm}$ and $9 \mathrm{~cm}$ (Figs. 1 upper, 2 upper, and 3 upper left).
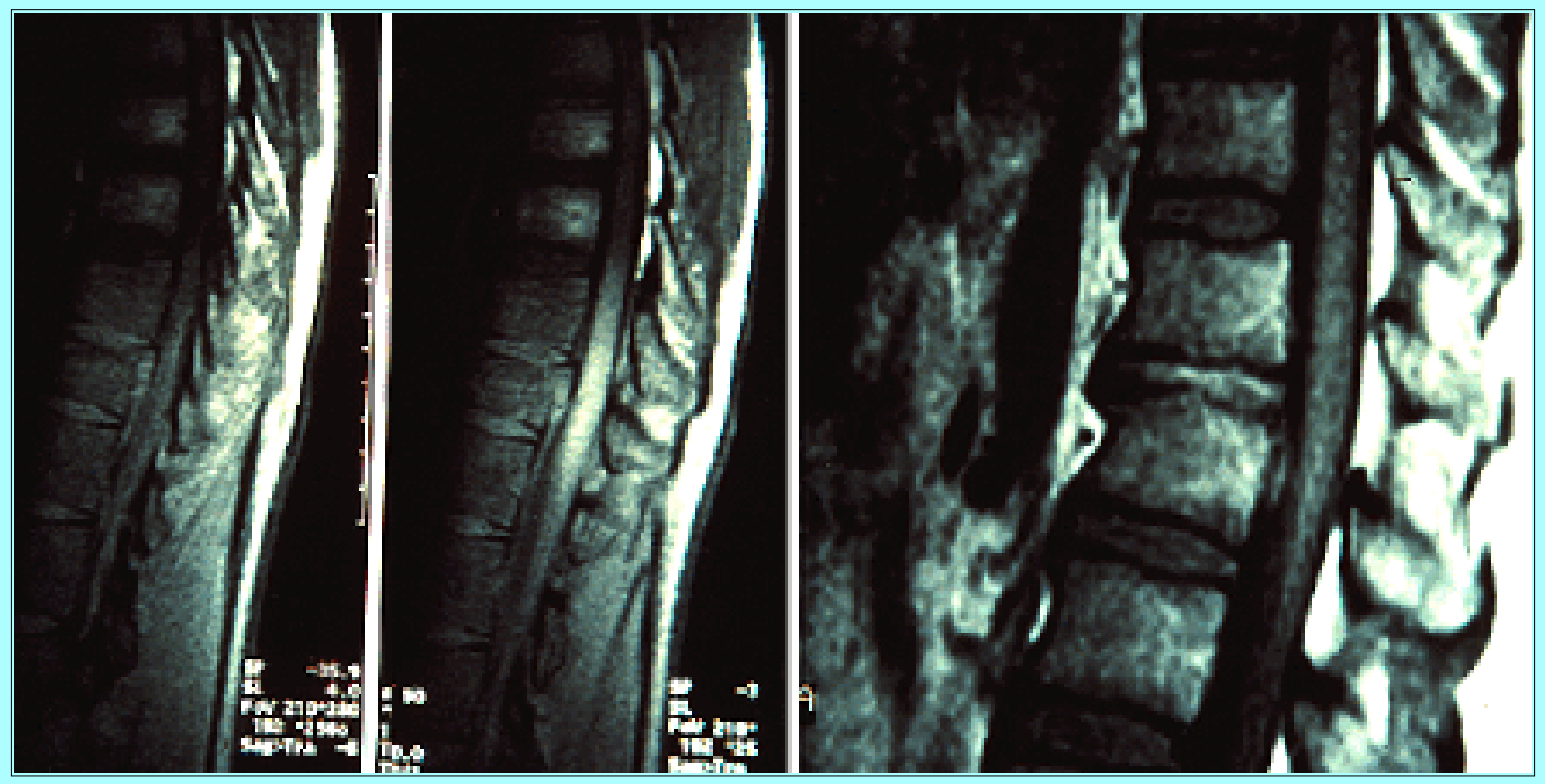

Fig. 1. Case 1. Left and Center: Sagittal $T_{1}$-weighted MR images obtained on the day of hospital admission. An isointense spinal epidural hematoma is seen anterior to the spinal cord. Right: Magnetic resonance image obtained on Day 9 postinjury reveals near-complete spontaneous resolution of the epidural hematoma. 


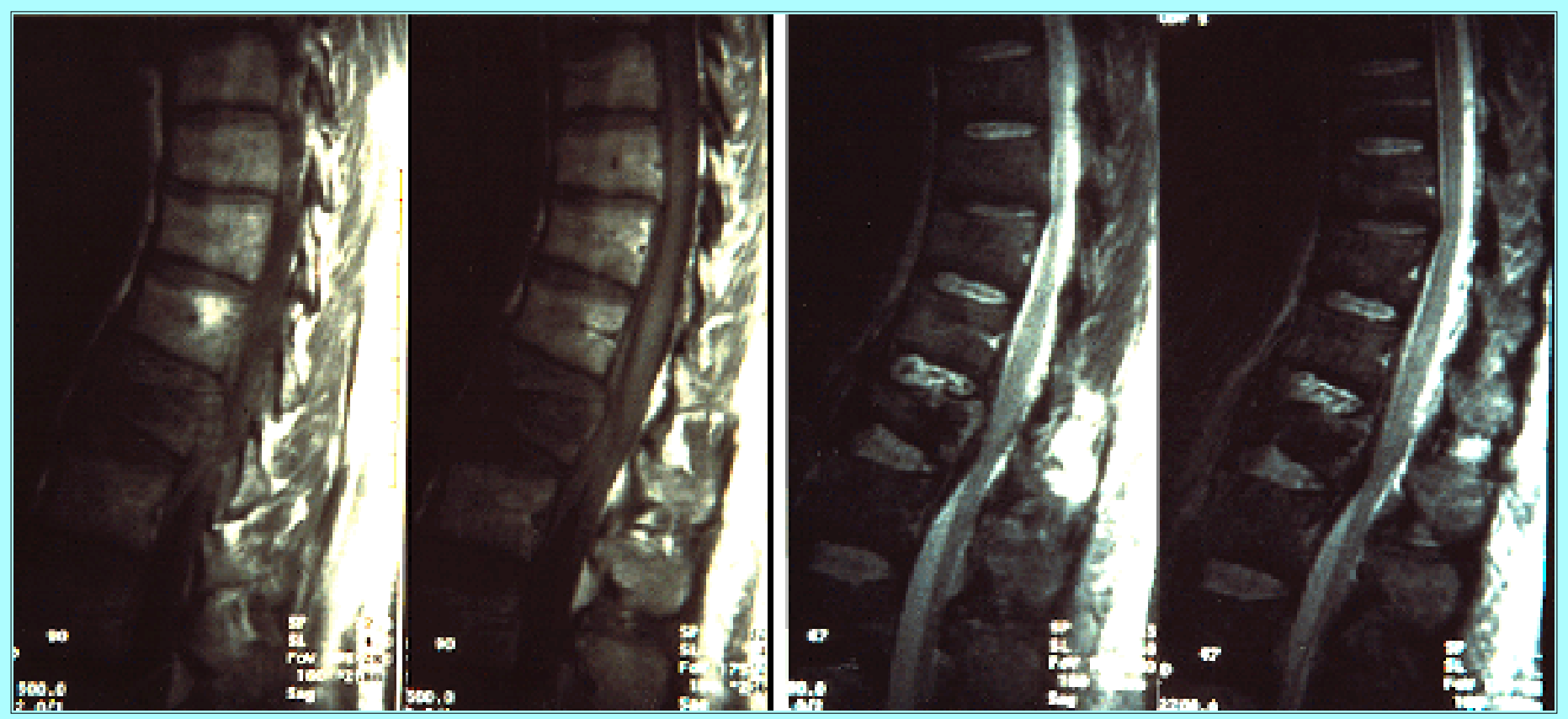

Fig. 2. Case 3. Magnetic resonance imaging studies. Left: Sagittal $\mathrm{T}_{1}$-weighted image obtained on the day of hospital admission. An isointense spinal epidural hematoma extending from T11-L2 levels is seen anterior to the spinal cord producing compression of the sac. This is the largest hematoma in our series. Right: $\mathrm{A} \mathrm{T}_{2}$-weighted image obtained on Day 10 after injury revealing resolution of the epidural hematoma.

No intramedullary signal abnormalities were seen. None of the patients exhibited neurological deterioration, and signs and symptoms normalized within 36 hours of admission. In light of each patient's fairly good neurological condition, a conservative approach was taken and steroid therapy initiated. Control MR imaging studies performed on Day 3 posttrauma revealed moderate reduction in size of SEH. Signal intensity changed to isointensity or slight hypointensity on $\mathrm{T}_{1^{-}}$and diffuse hypointensity on $\mathrm{T}_{2}$-weighted MR images (Fig. 3 upper right and lower left and right). 


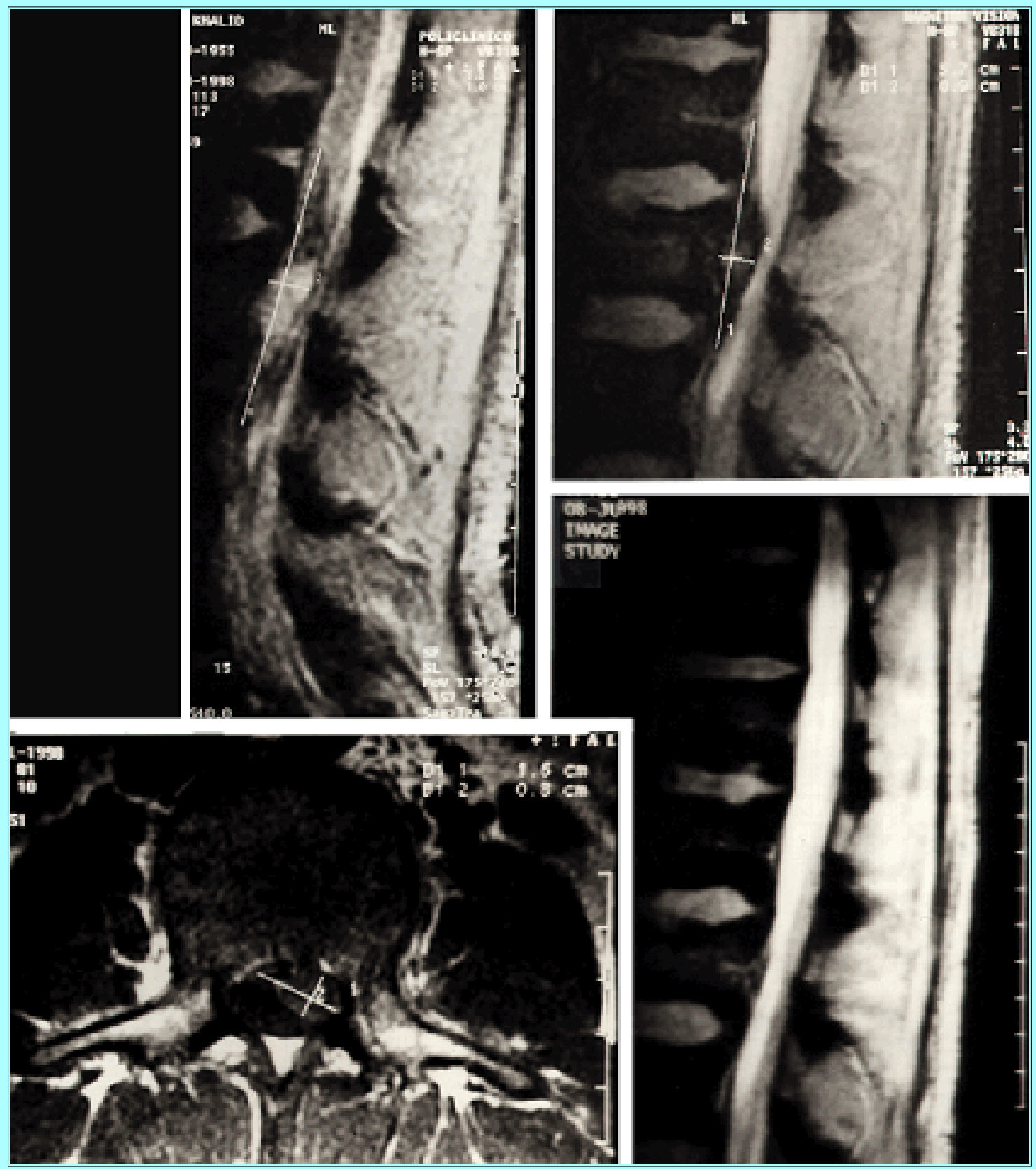

Fig. 3. Case 4. Upper Left: Gradient-echo two-dimensional (2D) $\mathrm{T}_{2}$-weighted MR images, sagittal view, obtained 3 hours postinjury revealing an anterolateral spinal epidural hematoma that extends from L2-L4 (length $6.5 \mathrm{~cm}$, thickness $1.0 \mathrm{~cm}$ ) with an inhomogeneous signal intensity. Upper Right: Same view as in upper left image, obtained on day 3 postinjury revealing a slight reduction in the hematoma size (length $5.7 \mathrm{~cm}$, thickness $0.9 \mathrm{~cm}$ ) with diffuse hypointense signal. Lower Left: Transverse (spin-echo $\mathrm{T}_{1}$-weighted) image obtained on Day 3 postinjury demonstrating the anterolateral right position of the lesion, which shows a signal isointensity. Lower Right: Sagittal view (gradient-echo 2D $\mathrm{T}_{2}$-weighted) image obtained on Day 10 postinjury demonstrating the spontaneous disappearance of the hematoma.

The SEH had completely resorbed by follow-up MR studies, which were performed on Day 8 in one case, on Day 9 in another, and on Day 10 in the remaining two patients (Figs. 1B, 2B, and 3D). At that time small focuses of residual high signal intensity on $\mathrm{T}_{1}$-weighted images consistent with clot lysis were also seen. 
All patients were discharged neurologically intact 2 weeks after trauma. Subsequently, at late follow-up clinical examination, their electromyographic and neurological examinations were normal.

\section{DISCUSSION}

We have had the opportunity to evaluate acute posttraumatic SEHs in four patients who have shown spontaneous resolution of their hematomas and associated symptoms. Although we do not generally recommend nonoperative treatment of traumatic SEH, we believe that with the increasing availability of 24-hour MR imaging services and use of MR imaging in spine-injured patients, a subgroup of patients will be identified who have mildly or minimally symptomatic SEH and in whom surgical decompressive procedures may not be necessary. Increased awareness of this problem should increase our index of suspicion for their recognition.

Traumatic SEHs associated with vertebral body or posterior arch fractures and/or ligamentous disruption[6] are a well-recognized but rare entity. They classically present in patients with a neurological deficit that progresses over a few hours interval after the injury. The clinical onset is generally characterized by radicular pain followed by symptoms of spinal cord compression; the neurological worsening may progress to para- or tethraparesis.[7] Exceptionally, Brown-Séquard syndrome or anterior cord syndrome have been reported.[4,5] Traumatic causes also include obstetrical birth trauma, lumbar punctures, postsurgical bleeding, and missile injuries. Although the true incidence of traumatic SEH is not precisely known, according to several series it can be estimated as ranging from 0.5 to $7.5 \%$. [2,7,19] Traumatic SEH may occur also in cases of minor trauma, without bone disruption, particularly in younger patients because of the greater elasticity of the spinal column, probably by a tearing action of epidural veins during acute disc disruption. $[4,19]$

Other causes have been described in association with SEH, such as coagulopathy, vascular malformation, and tumor.[14,16,22] However, in a substantial proportion of patients with SEH, these or other significant precipitating factors cannot be recognized.[18] Whereas a large body of literature exists regarding "nontraumatic" SEHs[16] and their surgical[11] or conservative[18] treatment, only a few reports have appeared in which the issue of management options for traumatic SEH is addressed. In a pre-MR imaging era literature review, Foo and Rossier[7] collected 38 such cases. Seventy percent of patients with fractures and 33\% of those with posttraumatic SEH without bony damage underwent laminectomy. Postoperative neurological recovery was observed in $38.5 \%$ and $88.9 \%$ of these two groups of patients, respectively. In the modern neuroimaging era, to our knowledge, there have been only three other reports on patients whose traumatic SEHs were managed conservatively.[4,13,19] All these patients presented with an incomplete neurological deficit after a relatively mild traumatic event, without spinal fractures, experienced a sustained spontaneous neurological improvement shortly after trauma, and recovered fully without surgery. In this respect, the patients in our series are relatively unique in that all of them suffered severe spinal trauma with vertebral fracture; on the other hand, their neurological deficits were minimal and improved rapidly after injury. The decision not to operate was based on their clinical presentation rather than their MR imaging findings.

With regard to the mechanism of SEH formation, there is no agreement in the literature on whether the origin of the bleeding is arterial or venous.[10,11] The rich spinal epidural venous network, constituted by valveless veins not protected against variation of abdominal or thoracic pressure,[16] is believed to be the major source of SEHs.[10] Pressure in the epidural veins, however, is lower than in the intrathecal compartment, and therefore venous bleeding cannot progress to causing significant compression of the 
dural sac. Limitation of hematoma extension within three vertebral levels in our series might be due to the venous origin of the bleeding; that is, the counterpressure exerted by structures in the spinal canal may have limited further progression of bleeding. The possibility of arterial bleeding that originated from the extensive network of epidural arteries should also be taken into consideration.[1,3] The origin of bleeding from the cancellous bone exposed by the fracture is important in cases of traumatic SEH. A history of trauma was a feature in all cases of our series. Although the integrity of the spinal canal was maintained, the vertebral bodies were fractured, and this probably contributed to the formation of the clots.

Spinal epidural hematomas are best treated as surgical emergencies, and recognition of symptoms and prompt differential diagnostic evaluation are essential in their management. Magnetic resonance imaging has now replaced myelography and computerized tomography scanning as the diagnostic procedure of choice, permitting complete investigation of this lesion. Multiplanar MR images scans visualize the relationship between the spine and spinal cord, as well as the extent of the hematoma and the degree of spinal cord compression.[14] Spinal cord decompressive surgery, by evacuation of hematoma through a laminectomy that extends over all levels where clot is located, should be performed promptly. Generally, the extent of neurological recovery is directly related to the promptness with which the hematoma is removed once neurological compromise begins. [7,8,11,14,15] However, the greater use of MR imaging within few hours after the traumatic event, also in mild-moderate spine trauma, will likely result in an increasing number of SEHs being revealed in patients who are either asymptomatic or only mildly symptomatic. The literature does not address the issues of prognosis and management in these patients.

The outcome of the patients presented here and the few cases with spontaneous remission of traumatic SEHs reported previously $[4,7,13,19]$ suggest that surgery may not be always necessary. The particular location of the SEH in the present series clearly influenced the favorable pattern of clinical presentation. High lumbar spine location, both for availability of intraspinal space and characteristics of vascular supply, allows for better tolerance of the extradural mass, as compared with the cervical or thoracic region, and the nerve roots of the cauda equina are relatively more tolerant to compressive mass lesions.[11,19]

What is the temporal profile of healing and what is the plausible mechanism of disappearance of the hematoma? Serial MR images demonstrated the time course of clot resolution, which was initiated by Day 3 and completed in all cases by Day 8 to 10 postinjury. The resorptive process could be related to the presence of the vast capillary network in the fatty areolar tissue of the epidural space, which normally facilitates rapid absorption of extraneous substances, but it could also be attributed to the spreading of the clot along the spinal canal or to the egress into the soft tissues surrounding the spine. We suggest that a MR imaging-monitored conservative management of traumatic SEH is legitimate in minimally symptomatic patients or in those in whom initial neurological deterioration is followed by early and sustained spontaneous recovery. For spine-injured patients with such a favorable clinical presentation who are at significant medical risk, a nonoperative management may indeed be the most appropriate approach.

\section{CONCLUSIONS}

Spinal epidural hematomas represent a neurosurgical emergency, and prognosis for recovery depends on the duration of neurological deficit prior to undergoing a decompressive laminectomy procedure and evacuation of the clot. The conservative treatment option should be reserved for use in exceptional 
situations when the patient shows minimal deficits, when neurological deterioration is followed by early and sustained spontaneous recovery, and when there are clear medical contraindications for surgery. The results of the present study confirm that nonsurgical treatment is feasible in a subgroup of minimally symptomatic patients who harbor moderate-sized SEHs. Although our experience shows a good spontaneous outcome for some patients with traumatic SEH, further studies are necessary to understand the real spectrum of nonsurgical treatment of such lesions.

\section{References}

1. Beatty RM, Winston KR: Spontaneous cervical spinal epidural hematoma. A consideration of etiology. J Neurosurg 61:143-148, 1984

2. Bruyn GW, Bosma NJ: Spinal extradural haematoma, in Vinken PJ, Bruyn GW (eds): Injuries of the Spine and Spinal Cord. Handbook of Clinical Neurology, Vol 26, Part 2. Amsterdam: North-Holland, 1976, pp 1-30

3. Clarke DB, Bertrand G, Tampieri D: Spontaneous spinal epidural hematoma causing paraplegia: resolution and recovery without surgical decompression. Neurosurgery 30:108-111, 1992

4. Crabbe DCG, Mendelow AD, Pharoh P, et al: Cervical spinal extradural haematoma causing a transient Brown-Sequard syndrome. J Neurol Neurosurg Psychiatry 55:239, 1992 (Letter)

5. Egido, Herrero JA, Saldana C, Jimenez A, et al: Spontaneous cervical epidural hematoma with Brown-Sequard syndrome and spontaneous resolution. Case Report. J Neurosurg Sci 36:117-119, 1992

6. Farias JP, Almeida Lima J, Lobo Antunes J: Subacute cervical epidural hematomas. Surg Neurol 42:414-416, 1994

7. Foo D, Rossier AB: Post-traumatic spinal epidural hematoma. Neurosurgery 11:25-32, 1982

8. Foo D, Rossier AB: Preoperative neurological status in predicting surgical outcome of spinal epidural hematomas. Surg Neurol 15:389-401, 1981

9. Frankel HL, Hancock DO, Hyslop G, et al: The value of postural reduction in the initial management of closed injuries of the spine with paraplegia and tetraplegia. I. Paraplegia 7:179-192, 1969

10. Groen RJM, Ponssen H: Vascular anatomy of the spinal epidural space: considerations on the etiology of the spontaneous spinal epidural hematoma. Clin Anat 4:413-420, 1991

11. Groen RJM, van Alphen HAM: Operative treatment of spontaneous spinal epidural hematomas: a study of the factors determining postoperative outcome. Neurosurgery 39:494-509, 1996

12. Kingery WS, Seibel M, Date ES, et al: The natural resolution of a lumbar spontaneous epidural hematoma and associated radiculopathy. Spine 19:67-69, 1994

13. Laissy JP, Milon P, Freger P, et al: Cervical epidural hematomas: CT diagnosis in two cases that resolved spontaneously. AJNR 11:394-396, 1990

14. Langmayr JJ, Ortler M, Dessl A, et al: Management of spontaneous extramedullary spinal haematomas: results in eight patients after MRI diagnosis and surgical decompression. J Neurol Neurosurg Psychiatry 59:442-447, 1995 
15. Lawton MT, Porter RW, Heiserman JE, et al: Surgical management of spinal epidural hematoma: relationship between surgical timing and neurological outcome. J Neurosurg 83:1-7, 1995

16. Lonjon MMC, Paquis P, Chanalet S, Grellier P: Nontraumatic spinal epidural hematoma: report of four cases and rewiew of literature. Neurosurgery 41:483-487, 1997

17. Miyagi Y, Miyazono M, Kamikaseda K: Spinal epidural vascular malformation presenting in association with a spontaneously resolved acute epidural hematoma. Case report. J Neurosurg 88:909-911, 1998

18. Pahapill PA, Lownie SP: Conservative treatment of acute spontaneous spinal epidural hematoma. Can J Neurol Sci 25:159-163, 1998

19. Pan G, Kulkarni M, MacDougall DJ, et al: Traumatic epidural hematoma of the cervical spine: diagnosis with magnetic resonance imaging. Case report. J Neurosurg 68:798-801, 1988

20. Saito S, Katsube H, Kobayashi Y: Spinal epidural hematoma with spontaneous recovery demonstrated by magnetic resonance imaging. Spine 19:483-486, 1994

21. Wagner S, Forsting M, Hacke W: Spontaneous resolution of a large spinal epidural hematoma: case report. Neurosurgery 38:816-818, 1996

22. Zuccarello M, Scanarini M, d'Avella D, et al: Spontaneous spinal extradural hematoma during anticoagulant therapy. Surg Neurol 14:411-413, 1980

Manuscript received November 17, 1998.

Accepted in final form December 17, 1998.

Address reprint requests to: Domenico d'Avella, M.D., Neurosurgical Clinic, Policlinico Universitario, via Consolare Valeria 1, 98100 Messina, Italy. email: davellan@ @ pharma.unime.it. 